\section{The effect of interval and number on masking with dot bursts*}

\author{
WILLIAM R. UTTAL $\dagger, \dagger \dagger$ \\ University of Michigan, Ann Arbor, Michigan 48104
}

Bursts of dotted visual noise can interfere with the recognition of dotted alphabetic characters during the period following the presentation of the character. The magnitude of this interference is a function of both the number of dots and the interval between the character and the visual noise burst. The nature of this interaction is explored in this paper and, on the basis of the results obtained, an attempt is made to clarify current notions of the period of interaction surrounding real time visual events.

Temporal discrimination capacities of the human perceptual system have been known for many years to be only modestly acute. Studies of the loss of temporal separateness, i.e., of perceptual simultaneity (Lichtenstein, 1961; Fraisse, 1966), of that elusive phenomenon, the psychological moment (Stroud, 1955; Allport, 1968), or of short-term visual storage (Averbach \& Coriell, 1961; Ericksen \& Collins, 1968) all essentially deal with the same problem: How are the finely divided moments of real time transduced into the far less highly resolved temporal units within our perceptual system? The experiments and interpretations dealing with this phenomenon have produced a conglomeration of perspectives and, at first glance, a family of somewhat incompatible findings.

Nevertheless, all of this work makes it clear that the ability to deal with time, as time, is substantially reduced in the visual system as one passes from the stimulus to the central neural event. We do not yet know the complete details of this loss; however, a central hypothesis, which may help explain experimental findings concerning simultaneity, sequential masking, and the psychological moment, is that there is a temporal dispersion of brief stimulus events into longer neural and subjective events. There is ample direct and indirect evidence that this temporal defocusing or slurring does occur, and that

*This work was supported in part by NSF Grant GB 12771 and in part by University of Michigan research funds. Substantial amounts of the writing and final revision of the paper were carried out at the University of Western Australia during a visit there. I am especially grateful for the exceptionally cordial hospitality of Professors Aubrey Yates and John Ross and Dr. Vincent Di Lollo, who made this visit so productive and pleasant.

$\dagger$ Address: 1042 M.H.R.I., University of Michigan, Ann Arbor, Michigan 48103.

$\dagger \dagger I$ am indebted to Jon Baron for the stimulating conversations we had with regard to the relevance of weighting functions to notions of the psychological moment. micromoments of real time in the world of the stimulus are extended into much more prolonged psychological and neurophysiological phenomena. Furthermore, and most important, it is also clear that during the prolongation there is no a priori reason to assume that all portions of the elongated percept are weighted equally with regard to their perceptual efficiency. Experiments on visual masking (Kinsbourne \& Warrington, $1962 \mathrm{a}, \mathrm{b}$; Schiller \& Smith, 1965; Kahnemann, 1968; Uttal, 1969a) suggest that there is, quite to the contrary, a weighting of the efficiency of any given masking stimulus, depending upon how close it is to the masked stimulus.

Herein lies the critical issue of the controversy surrounding the notions of psychological moments and perceptual simultaneity. The integrative mathematics which is often used to analyze these events is generally unable to distinguish the phase relations of the integrating period or moments (or whatever other name has been applied to the period of interaction surrounding the stimulus). Thus, like almost any correlation technique, the consolidation of experimental data into a simple statistical measure means a loss of some of the original information. Unfortunately, the phase information lost when one pools data or performs a correlation is the critical dimension in the controversy over discrete discontinuous moments and continuously sliding moments. From this point of view, all experiments which pool data over many trials must be considered ill-equipped to resolve the controversy.

What is meant by these two terms-the discrete discontinuous moment and the continuous sliding moment? Both refer to an integrating period in which information about the temporal order of stimuli is lost. This term "moment" was probably first used by Stroud (1955) when he described his ideas of a discrete and discontinuous interval in which order information is lost, but the general notion of an integrating period has been used by many other authors. We use the term here in the more general sense, denoting the temporal interval between two stimuli in which they interact in one way or another. Simply stated, the two kinds of moment describe different ways in which the degree of interaction may be weighted as a function of the size of the interval between the two stimuli. The notion of the continuously sliding moment is characterized by the assumption that a region in which interaction occurs surrounds every stimulus. This region is weighted differentially so that the closer one stimulus is to another, the greater will be the interaction. This idea is an analog of the weighting function of the lateral inhibitory interaction described by Hartline, Wagner, and Ratliff (1956), but it operates in time rather than space. We have called it the sliding window hypothesis.

It should be noted that the sliding window hypothesis is very similar to the notions of the temporal summation model of masking proposed by Eriksen and Hoffman (1963) and Kinsbourne and Warrington (1962a, b), and further developed by Kahnemann (1967), although the notion that weighted effectiveness of interaction is a function of the temporal distance from the stimulus is an added parameter not considered by some of these workers. The sliding window notion also has great similarity to the term "spread function" suggested by Baron and Krantz ${ }^{1}$; one occasionally also hears the related terms "temporal receptive field," "psychological time quantum," and "sensory trace." Sperling and Sondhi's (1968) "impulse function" and Shallice's (1967) "moving average" also seem to denote similar ideas.

Another important consideration is that while we will often refer to the window as a region surrounding the character, implying that it occurs both prior to and following the character, and while we often measure the function in this manner, the interaction is not truly bilateral. A period follows a stimulus event in which the trace of that event is capable of interacting with another stimulus event. Thus, a leading mask appears as a region of interaction preceding a test stimulus if the criterion is the effect on the test stimulus; yet, clearly, what is really meant is the persistent effect of the leading noise on the trailing stimulus. The region of interaction surrounding a stimulus is, therefore, the period following it plus the period following any other stimulus which may have preceded it. This simplistic notion is weakened by the fact that most masking situations, while similar, do not produce identical behavior in the forward and 
backward directions (Schiller \& Smith, 1965; Uttal, 1969a). This discrepancy is yet to be explained.

The usual alternative formulation, the discontinuous discrete moment notion, assumes that the weighting function is not differentially weighted and sliding with the stimulus but is, rather, rectangular and not timelocked to the stimulus. From this point of view, any two events which occur within the same rectangular summation period would interact equally strongly, regardless of the interval between the two. The continuous functions of interval obtained in the several experiments mentioned above would therefore be produced by the synthesis of the results of randomly placed periods of interaction over the many trials of any given experiment.

The random dot procedures which we have used in a number of earlier studies (Uttal, 1969a, b; 1970a, b, c), both in the form we called dynamic visual noise (DVN) and in the static random pattern, provide an opportunity to explore this sort of problem in an unusually quantitative fashion. Dots may be dispersed over elongated periods of time or concentrated at specific instants at specific delays. As previously noted, such a precisely defined stimulus provides an unusually effective assay technique for exploring the interactions of sequential visual events. However, it does not provide a better route for answering the question of continuity vs discreteness than does any other technique using pooled data.

As we have said, the main criterion which distinguishes continuously sliding moment from the discrete discontinuous moment is whether the period of interaction is rectangular and unrelated to the stimulus or whether it is a continuous, differentially weighted function surrounding and time-locked to the stimulus event. There is only one experiment (Allport, 1968) with which we are familiar which specifically tests this issue; all others, to the best of our present knowledge, are ambiguous. This elegant work of Allport is necessarily in the form of a demonstration rather than a statistical study using pooled data. Allport concludes that there is no support for the latter of the two alternatives mentioned above, which he refers to as the "discrete moment hypothesis" (following Stroud, 1955). He believes that his results support the notion which he calls the "continuous or traveling moment hypothesis."

In retrospect, we no longer feel that our experiments provide a conclusive argument for the sliding moment hypothesis because statistical pooling of data loses phase information. However, we still believe that the perspective of modern neurophysiology renders implausible the notion of the randomly placed, rectangular, and discrete moment. Allport's carefully conceived experiments add further credence to the alternative point of view: that each stimulus event, no matter how brief, is surrounded by a temporal region in which it can interact with other stimuli. Our earlier experiments, as well as those of many other workers in the field, suggest further that this region of interaction is weighted by a function which is dependent upon the interval between the two stimuli. We further assume, although this is subject to doubt, that this region of interaction slides continuously along the real-world time line. Questions of simultaneity arise because of indiscriminably small differences in the weights of sequential eyents.

These, then, spelled out in detail, are the basic premises of the following work. In this light, some possible experimental questions become nonsensical and others become empirical issues eminently susceptible to quantitative measurement. The search for the phase relationships of rectangular and discrete moments, from this point of view, is a vain search for a nebulous chimera. At the very best, we will always be bounded by the problems of data pooling; and at the very least, discrete moment may simply not exist. On the other hand, if we accept the notion of the sliding moment as a conceptual model and as an initial axiom, we can make meaningful statistical measurements of temporal interaction.

We report here the results of a set of experiments in which we measured the interaction between sequential stimuli, using the same visual pattern recognition technique described in our earlier reports. This technique is analogous to a signal-to-noise paradigm more commonly found in auditory experiments. Dotted alphabetic characters serve as the signal and a burst of random dots as the noise. The interaction is measured by determining what percentage of the total number of characters is correctly identified as a function of the interval size and the density of the visual noise. The random dot patterns which constitute the noise are plotted in rapid bursts at various intervals and densities following the presentation of a dotted character. This technique is used rather than the DVN technique used earlier. It is somewhat like techniques of French (1954) and Eriksen and Collins (1967). It is thus capable of providing the basic data to describe the weighting functions of the interaction between the sequential signal and noise patterns. An additional experiment was performed to determine what composition rules, if any, emerge when two bursts of random dots are presented at varying intervals following the character.

It is important to emphasize that while the experimental paradigm of backward masking is common to these experiments and to several other of the cited studies, it is now believed that dot masking assays, in part at least, substantially different mechanisms than those elicited by masking with continuous figures or flashes of light.

\section{METHOD}

\section{Subjects}

Six to eight Ss provided the data for each point plotted in our experimental graphs. Unfortunately, owing to their academic schedules, the group of Ss used was not the same throughout the course of the several experiments of this study. A comparison of absolute levels of performance of each group showed some small intergroup differences. The differences appear to be a function of the type and number of training sessions given to each group (a gradual increase in the difficulty of the stimulus materials apparently led to a higher overall score at later stages of the Ss' participation than did an abrupt increase.)

\section{Apparatus and Procedures}

The apparatus used in this experiment has been described in detail in reports of earlier studies (Uttal, 1969a, b; 1970a, b, c). Briefly, Ss observed dot patterns plotted on an ultrashort-persistence cathode ray tube (CRT) having a P15 phosphor designed to decay to $.1 \%$ of its initial brightness in about $50 \mu \mathrm{sec}$. The distance from the display to the bridge of the S's nose was 14 in., defining visual angles of 4 by $3 \mathrm{deg}$ for the character and 9 by $6 \mathrm{deg}$ for the dotted noise field. His head was restrained by a forehead rest. The plotting of the points constituting both the visual noise and the character was under the programmed control of a digital computer whose output signals were transformed by appropriate digital-to-analog converters. The S's responses were spoken over an intercom from his light- and soundproof isolation chamber to a transcriber who keyed these single alphabetic characters into a standard keyboard connected to the computer, where they were analyzed immediately.

The S's task in each of the experiments reported here was to identify the randomly selected 1 of 24 possible alphabetic characters $(O$ and $Q$ being omitted for obvious reasons). The test character was masked to a greater or lesser degree by static bursts of randomly placed dots. The 


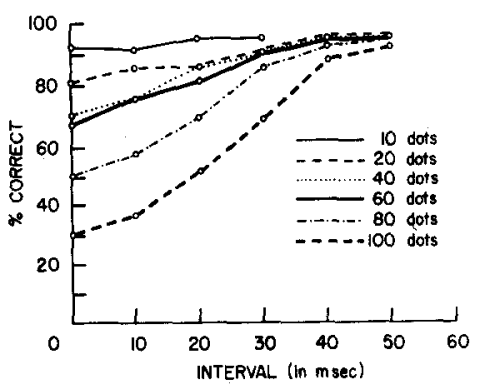

Fig. 1. The results of the first experiment are plotted as a function of the interval between the presentation of the character and the single noise burst. The curves are parametric in the number of dots contained in the noise burst.

number and position of the dots and the interval between the character and the following dots were also varied by the program. The term "static burst" refers to a situation in which all of the dots of the visual noise are plotted as rapidly as possible at some predetermined interval following the character. The computer used in this experiment was fast enough to plot 100 dots at random locations in about $3 \mathrm{msec}$. No attempt was made during the 3-msec period to actually calculate the random positions; this calculation was performed during each preparatory prestimulus period, and the random locations were stored in a table for use during the stimulus generation. A table-scanning algorithm was then manipulated to facilitate maximum speed of point plotting. In both experiments reported here, the bursts of visual noise always followed the presentation of the character. The paradigm used, therefore, is a backward masking situation, and the weighting functions obtained are only valid for the retroactive effect.

Two Ss ran together for a 1 -h session. Each $\mathrm{S}$ participated for $1 / 2 \mathrm{~h}$ as an $\mathrm{O}$ and $1 / 2 h$ as a transcriber at the computer input keyboard. The results of each trial were shown, on a computer-controlled alphanumeric television display, to the transcriber. He fed back the name of the correct character if, and only if, a mistake had been made. The paradigm for the presentation of each trial was the same as used previously. A "ready" signal, consisting of a single steady dim dot, appeared at the top of the CRT when the computer was prepared to present a trial. The $S$ pressed a hand-held pushbutton when he was ready, releasing the stimulus train. This train consisted of a constant blank interval following the instant at which the ready signal was extinguished, the plotting of the character itself in a period of about $1 / 3 \mathrm{msec}$, a variable delay, and then the static noise burst of varying density. In the second experiment reported below, a variable delay and a second static noise burst followed the first delay and masking burst.

Experiment 1 was designed mainly to determine the masking effectiveness of static noise bursts as a function of the interval between the character and the noise as we varied the number of the masking dots. Intervals of $50,40,30,20$, 10 , and $0 \mathrm{msec}$ were used as the independent variable except for 10 and 20 dots (see below), where performance had reached zero noise levels at 30 and $40 \mathrm{msec}$, respectively. The intervals were held constant each day but presented in descending order as indicated on successive days. The experiment was done parametric in the number of dots in the burst. Densities of $10,20,40,60,80$, and 100 dots were used. Each data point plotted in Fig. 1 is the pooled result of all Ss on a single day and represents the average recognition score for 2,000-2,500 trials.

Experiment 2 was designed to elucidate what higher order effects, if any, would result if two, rather than one, static noise bursts were used as the masking stimuli. Three different conditions were run at each of four of the noise densities mentioned above: $40,60,80$, and 100 dots. In each of these three conditions, two static bursts were presented, each at a delay following the character. The pattern is defined by a three-number coding scheme. For example, 20-30-100 would indicate a masking pattern in which two bursts, each containing 100 dots, were presented in such a way that the first one began 20 msec after the character and the second one began $30 \mathrm{msec}$ after the character. A nominal interval of 10 msec separated the two masking bursts in this case. Because of the algorithm used for the computation of delays and the nontrivial duration of the first masking burst itself (about $3 \mathrm{msec}$ ), the second burst, however, always started about $3 \mathrm{msec}$ after the indicated time.

Data for this experiment were collected by use of essentially the same design used in Experiment 1. Each day a given masking condition was defined and held constant while the alphabetic characters were presented in random order. All data from all Ss were pooled on each day to give a single point representing, on the average, about $2,000-2,500$ trials. The experimental conditions used were $20-30-60,10-30-60$, $10-20-60,20-30-80,10-30-80,10-20-80$, 20-30-100, 10-30-100, and 10-20-100.

A brief supplement to this second experiment was then performed in which a single burst of noise dots, equal in number to the sum of the number of dots presented in hoth masking bursts of the

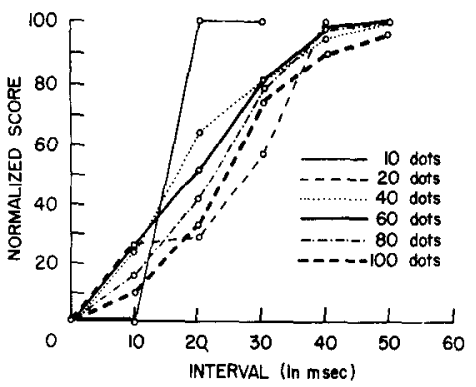

Fig. 2. The data of the first experiment are normalized as described in the text and plotted to show the general constancy of the course of the performance decline as the interval between the character and the single noise burst decreases. The only deviant points are for the extreme condition in which only 10 dots were used. The curves are parametric in the number of dots.

previous part of the experiment, was presented at a delay of $20 \mathrm{msec}$. These data were, therefore, comparable to the average delay of the two-burst situation when the first delay was equal to $10 \mathrm{msec}$ and the second was equal to $30 \mathrm{msec}$.

\section{RESULTS}

The results of Experiment 1 are plotted in Fig. 1. Character recognition, as indicated on the vertical coordinate in terms of a percent of the total number of presented characters which were correctly identified, while near perfect at intervals greater than $50 \mathrm{msec}$, decreases monotonically as the interval between the character and the static noise burst decreases. There does appear to be a slight decline in the rate of decrease at the shortest interval, and the curve does flatten out, suggesting a gentle S-shaped or sigmoidal form for each curve. These same data may be replotted in another interesting manner. Instead of plotting absolute scores, we have replotted the data in Fig. 2. The data are normalized so that the vertical coordinate has been changed to a percentage of the maximum decline in recognition scores for each $\mathrm{N}$. Thus, the bottommost points for all six $\mathrm{N}$ values $(10$, $20,40,60,80$, and 100 ) have been anchored together. Ninety-five percent was considered to be the maximum possible score. Each point was plotted according to the following relation: $\mathrm{NS}=$ $\% \mathrm{C} /\left(95-\mathrm{M}_{\mathrm{n}}\right)$, where NS is the normalized score, $\% \mathrm{C}$ is the percentage of the total number of presented characters which were correctly identified at that particular interval, and $M_{n}$ is the minimum recognition score for a given number of dots (i,e., the score at $0 \mathrm{msec}$ ).

When the data are plotted in this fashion in Fig. 2, it can be seen that, with the 


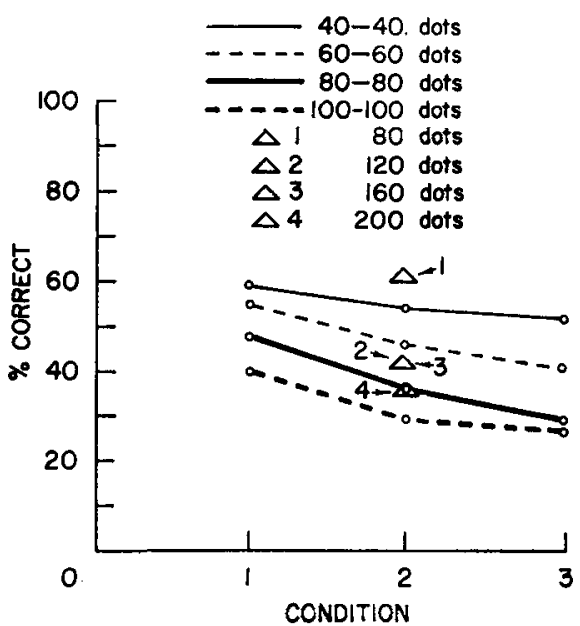

Fig. 3. The results of the second experiment are plotted as a function of the conditions described in the text. Condition 1 defines intervals of $20 \mathrm{msec}$ between the character and the first noise burst and $30 \mathrm{msec}$ between the character and the second noise burst. This has been coded as 20-30-n (where $n$ is the number of dots in each of the two masking bursts). Condition 2 is for the $10-30-n$ case and Condition 3 is for the $10-20-n$ case. The four curves are parametric in the number of dots ( $n$ ) in each burst. The four free points indicated by $\Delta$ are for the four conditions of the supplement to the second experiment. An interval of $20 \mathrm{msec}$ (the average of the intervals of Condition 2) was used for a single burst of $80,120,160$, or 200 dots.

exception of the extreme case in which there were only 10 masking dots, the curves for the different values of $\mathrm{N}$ are very nearly of the same shape. There are some differences; as $\mathrm{N}$ increases the decrease in the normalized score occurs slightly faster. For example, at $20 \mathrm{msec}$ there appears to be about a $20 \%$-point difference between 60 and 100 dots. Nevertheless, the course of the decrease in performance is generally of the same form for all values of $\mathrm{N}$.

The results of the second experiment and its supplement are shown in Fig. 3. These results exhibit no surprising features; the main effect is an increase in masking effect as the two noise bursts are brought closer to the stimulus character or as the density of the masking dots increases. The fact that the lines connecting the points for each of the three conditions for each dot numerosity are concave upward indicates that the differential effect of reduced interval is lessened as the absolute level of masking increases. This is confirmation of the slight flattening of the data in the first experiment and of the notion that the overall function is sigmoidal.

The free dots, representing the results of the supplementary experiment, in which the sum of the dots in the two bursts was presented at the average value of their delay, fall closely to the results for the corresponding two-burst condition, indicating that there is probably no important difference between the two- and single-burst condition.

Another interesting replotting of the data is presented in Fig. 4, in which some of the conditions of both experiments are extracted to elucidate the effect of dot numerosity per se. Figure 4 summarizes the results for all data with a delay of $20 \mathrm{msec}$ plotted as a function of the number of dots contained in the noise burst. This figure shows very clearly the sigmoid nature of the curve as dot numerosity is varied, and the almost linear central region in which the maximum differential effects of dot numerosity are obtained.

\section{DISCUSSION}

This examination of the effectiveness of bursts of random noise dots as inhibitors of alphabetic character recognition results in data which define a surface in a tridimensional space of interval, noise-dot numerosity, and performance score. This surface is gently sigmoidal along both of its major defining dimensions. As we have seen, in general, increasing the number of dots increases the masking more for central values than for either very small or for very large dot numerosities. Similarly, increasing the effectiveness of the noise by shortening the interval between it and the character is more effective for central values than for the extremes. Intuitively, such a result is not too surprising along the numerosity dimension; with a small number of dots, an increase in the number of masking dots would presumably continue to produce a situation in which the highly recognizable features of the character would still be expected to predominate. A few additional dots would thus have only a small effect. Furthermore, at the other end of the numerosity continuum, the masking effect is so great that little additional decrease might be expected as the number of masking dots increases. It is, rather, in the region of moderate numbers of masking dots that an additional few dots have their most substantial differential effect.

The sigmoidal shape of the surface reflects some further specific features of the weighting distributions. The fact that there is less difference in almost all cases between the 0 - and $10-\mathrm{msec}$ than between the 10 - and $20-\mathrm{msec}$ intervals suggests that there is a relatively flat top on the weighting curve describing the region of interaction or "moment" following the test character. It is clear (proceeding under the assumption that these regions of

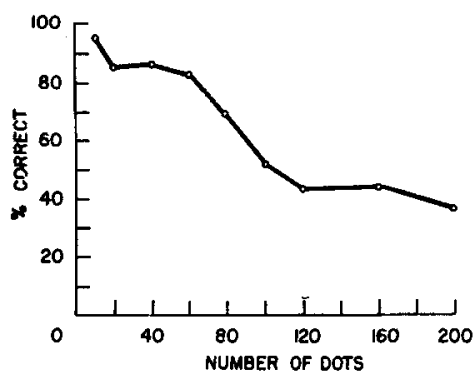

Fig. 4. Data are summarized from Figs. 1 and 3 to display the effect of dot numerosity on the recognition threshold. All of these data result from conditions in which the interval between the character and a single burst of visual noise was 20 msec.

interaction are sliding along the real-time axis and that our technique is adequately measuring their shape) that the region is neither rectangular nor very sharply peaked. Rather, it is a broad temporal region $80-100 \mathrm{msec}$ (twice the size of the backward portion we have measured here) in duration and varies considerably in its weighting over its course.

The data reported here and summarized in Fig. 4 are descriptive. They do not, however, contribute to our understanding of the mechanism underlying the $S$ 's performance in this task. We may speculate that this might possibly be a sort of brightness discrimination task in which the contrast between the fading dots of the character and the dots of the noise is the key variable used in discrimination. The data presented here may be representative of a short-term storage somewhere in the visual system, but one that certainly does not operate on an off-on basis. According to these results, the trace gradually fades, as reflected in the increase in performance as the interval between the noise burst and the character increases. On the other hand, the decrease in performance as the number of dots varies is not a measure of the decay of the visual trace as much as it is a measure of the ability of the entire visual system to process the information contained in the character and masking dots.

Many of the effects reported here and in previous papers in this series indicate that the effects observed are primarily due to a confusion of the dots of the noise with the dots of the character. Earlier work (Schiller, 1965; Uttal, 1970c) also confirmed the notion that this confusion is mediated by central neural mechanisms since the noise could be introduced through the eye opposite to that in which the character was presented yet mask as effectively as monoptic or binocular presentation. 
It had been originally intended in the design of this experiment to use the weighting functions obtained to develop a transformation model which would allow prediction of the effects of dynamic visual noice (DVN) reported in earlier experiments. The general notion was that there should be some composition rules which would allow the weighting values to be used as coefficients to explain how the psychological macromoments were synthesized from real-time micromoments. In spite of the fact that the supplement to the second experiment indicated that there were no major nonalgebraic effects which would lead to a major difference between one burst with $n$ dots placed at the average interval of two bursts with $\mathrm{n} / 2$ dots, and the two-burst situation, it was quite clear that there was no simple way in which one could predict the distributed effects of 100 dots from the local effectiveness of 10 groups of 10 dots individually presented at discrete intervals. This same limitation is evidenced by the sigmoidal nature of the data. The addition of 10 dots to a noise burst will have clearly different effects depending upon how many dots are already present in that burst and the performance level already defined by the interval. The composition rules which govern the slurring of visual moments, therefore, probably are very complex and, at least in the present context, insufficient evidence is available to develop a rigorous model.

These data are presented in an attempt to state more precisely the general notion that real time micromoments are converted into psychological macromoments by physiological mechanisms whose behavioral and functional characteristics can be described even if they cannot be anatomically identified. Our interpretation of these data is that they represent measures of the shape of the temporal slurring which has led to such notions as the psychological moment or visual simultaneity; thus they reflect some characteristics of the human short-term visual storage mechanism. It is important to note, however, as pointed out previously, that this region of temporal interaction is not one in which the confusion is complete or even constant over its whole course. Rather, a differentially weighted effectiveness is assignable to each interval between character and noise burst. These weighting values define a probabilistic interaction as a function of interval. The idea that this "moment" is continuously sliding along a real time axis and is unequally weighted over its course makes it a concept different from that of the discrete moment described by Stroud (1955) and others.

The characteristics of this region of interaction, as measured in the dot-masking experimental paradigm used in the present study, can be summarized in the following list: (1) Masking increases monotonically with noise dot density. (2) The masking function is sigmoidal as a function of noise dot density, with points of inflection at approximately 60 and 120 dots. Between these two values the effect of dot density is almost linear; in this central region it displays its maximum differential values. (3) Masking increases monotonically as the interval between the character and the mask decreases. (4) The masking function, as a function of interval, is also sigmoidal with points of inflection at about 10 and $40 \mathrm{msec}$. Between these two values, this curve is also approximately linear and displays its maximum differential values.

\section{REFERENCES}

ALLPORT, D. A. Phenomenal simultaneity and the perceptual moment hypothesis. British Journal of Psychology, 1968, 59, 395-406.

AVERBACH, E., \& CORIELL, A. Short term memory in vision. Bell System Technical Journal, 1961, 40, 309-328.

EFRON, R. The duration of the present. Annals of the New York Academy of Sciences, 1967, $138,713-729$.

ERIKSEN, C. W. \& COLLINS, J. F. Some temporal characteristics of visual pattern perception. Journal of Experimental Psychology, 1967, 74, 476-484.

ERIKSEN, C. W., \& COLLINS, J. F. Sensory traces versus the psychological moment in the temporal organization of form. Journal of Experimental Psychology, 1968, 77, 376-382.

ERIKSEN, C. W., \& HOFFMAN, M. Form recognition at brief durations as a function of adapting field and interval between stimulations. Journal of Experimental Psychology, 1963, 66, 485-499.

FRAISSE, P. Visual perceptive simultaneity and masking of letters successively presented. Perception \& Psychophysics, 1966, 1. 285-287.

FRENCH, R. S. Pattern recognition in the presence of visual noise. Journal of Experimental Psychology, 1954, 47, 27-31.

HARTLINE, H., WAGNER, H., \& RATLIFI, $r$,
Inhibition in the eye of Limulus. Journal of General Physiology, 1956, 39, 651-673.

KAHNEMAN, D. An onset-onset law for one case of apparent motion and metacontrast. Perception \& Psychophysics, 1967, 2, 577-584.

KAHNEMAN, D. Method, findings, and theory in studies of visual masking. Psychological Bulletin, 1968, 70, 404-425.

KINSBOURNE, M., \& WARRINGTON, E. The effect of an after-coming random pattern on the perception of brief visual stimuli. Quarterly Journal of Experimental Psychology, 1962a, 14, 223-234.

KINSBOURNE, M., \& WARRINGTON, E. Further studies on the masking of brief visual stimuli by a random pattern. Quarterly Journal of Experimental Psychology, 1962b, 14, 235-245.

LICHTENSTEIN, M. Phenomenal simultaneity with irregular timing of components of the visual stimulus. Perceptual \& Motor Skills, $1961,12,47-60$.

SCHILLER, P. Monoptic and dichoptic visual masking by patterns and flashes. Journal of Experimental Psychology, 1965, 69, 193-199.

SCHILLER, P., \& SMITH, M. A comparison of forward and backward masking. Psychonomic Science, 1965, 3, 77-78.

SHALLICE, T. Temporal summation and absolute brightness thresholds. The British Journal of Mathematical \& Statistical Psychology, 1967, 20, 129-162.

SPERLING, G., \& SONDHI, M. M. Model for visual luminance discrimination and flicker detection. Journal of the Optical Society of America, 1968, 58, 1133-1145.

STROUD, J. The fine structure of psychological time. In H. Quastler (Ed.), Information theory in psychology. Glencoe, Ill: The Frce Press, 1955. Pp. 174-207.

UTTAL, W. Masking of alphabetic character recognition by dynamic visual noise. Perception \& Psychophysics. 1969a, 6. 121-128.

UTTAL, W. The tharacter in the hole experiment: Interaction of forward and backward masking of alphabetic character recognition by dynamic visual noise (DVN). Perception \& Psychophysics, 1969b, 6, 177-181.

UTTAL, W. Masking of alphabetic character recognition by ultrahigh-density dynamic visual noise. Perception \& Psychophysics, 1970a, 7. 19-22.

UTTAL, W. Violations of visual simultaneity. Perception \& Psychophysics. 1970b, 7. 133-136.

UTTAL, $W$. On the physiological basis of masking with dotted visual noise. Perception \& Psychophysics, 1970c, 7, 321-327.

1. J. Baron and D. communication, 1970.

(Accepted for publication October 30, 1970.) 